\title{
Effets d'une réduction de l'apport protéique durant la gestation sur le métabolisme maternel et l'évolution de la composition corporelle au cours du cycle de reproduction chez la truie
}

\author{
P.H. DUEE (1), B. DESMOULIN, M. ETIENNE et G. DURAND * \\ avec la collaboration technique de Régine Calmes, Eliane PENOT, \\ Michèle Serezat, Christiane Vachot, F. Cointepas, P. Peiniau et J. Lebost
}

I.N.R.A., Station de Recherches sur l'Elevage des Porcs, Centre de Recherches de Rennes, F 35590 L'Hermitage

* I.N.R.A., Station de Recherches de Nutrition,

Centre de Recherches zootechniques, F 78350 Jouy-en-Josas

Résumé

Une expérience a été entreprise sur 58 truies de race Large White réparties, à la puberté, en deux lots ne différant que par l'apport protéique $(10$ ou 13,5 p. 100 de matières azotées). Dans chaque lot, les truies sont affectées à l'une des quatre situations suivantes : truies non gravides alimentées dans les mêmes conditions que les truies gravides et abattues 114 jours après la puberté ; truies saillies à la puberté et abattues dans les 48 heures après la parturition, au $21^{\mathrm{e}}$ jour de lactation ou 8 jours après le sevrage. Après leur mise bas, les animaux reçoivent le même régime (à 13,5 p. 100 de matières azotées).

L'administration du régime à 13,5 p. 100 de matières azotées durant la gestation accroît le gain de poids des truies d'environ 16 p. 100, correspondant à un accroissement du gain net (23 p. 100) sans modification du poids de portée à la naissance. Chez les truies non gravides, l'augmentation correspondante du gain de poids est moindre $(11 \mathrm{p}$. 100). Leur bilan azoté, établi après 80 jours d'expérience, n'est d'ailleurs pas modifié. Chez les truies gravides au contraire, la quantité d'azote retenu augmente, avec l'apport protéique, de 9,3 à 12,0 g par jour. En fin de gestation, l'aminoacidémie libre des truies gravides est caractérisée par une élévation de certains acides aminés non indispensables (Asp, Gln, Gly, Ala), associée, au taux protéique le plus bas, à une diminution des teneurs en quelques acides aminés indispensables (Leu, Val, Lys).

L'activité métabolique du tissu adipeux sous-cutané (activité de la lipoprotéine lipase, lipogenèse à partir du glucose) diminue durant la seconde moitié de la gestation. A la parturition, l'augmentation de la consommation azotée se traduit chez les truies gravides par un dépôt musculaire plus important (découpe parisienne de la carcasse, densité du «rein» et du jambon, surface de noix de côtelette, épaisseur de lard). Ce degré de musculature plus élevé ne se retrouve pas chez les truies non gravides.

En lactation, les performances des animaux (production laitière, composition du lait, croissance de la portée, bilan azoté) sont comparables qu'ils aient reçu 10 ou 13,5 p. 100 de protéines pendant la gestation. L'activité métabolique du tissu adipeux sous-cutané reste peu élevée : il existe d'ailleurs une relation linéaire inverse entre l'activité de la lipoprotéine lipase du tissu adipeux sous-cutané et la production laitière à 10 jours de lactation. La perte de poids, qui caractérise cette période, se traduit principalement

(1) Demandes de tirés à part à adresser à : P.H. DuÉE, Centre de Recherches sur la Nutrition du C.N.R.S., 9, rue J.-Hetzel, F 92190 Meudon-Bellevue. 
par une mobilisation importante des masses musculaires (diminution de 25 p. 100 de la surface de noix de côtelette). Après le sevrage, l'effet du taux protéique de gestation sur la composition corporelle des mères subsiste, ce qui suggère l'existence d'une relation entre la quantité de protéines consommée durant la gestation et l'état nutritionnel des animaux après un cycle de reproduction.

\section{Introduction}

La relation qui existe entre l'état nutritionnel des truies et leur productivité (nombre et poids des porcelets au sevrage) est admise mais encore mal expliquée. Si une réduction globale des apports nutritionnels ou une alimentation déséquilibrée appliquée lors d'une seule gestation n'a que peu d'effet sur le niveau de productivité, ce n'est plus le cas lorsque la diminution des apports nutritionnels porte sur une période plus longue. L'ampleur des variations pondérales durant le cycle de reproduction en est alors modifiée et peut expliquer l'effet à plus long terme d'une réduction des quantités consommées par la mère sur la productivité (DuEE, 1976).

Dans un travail préliminaire (DueE, Treil \& Camous, 1980), nous avons montré que la réduction de l'apport protéique durant la gestation chez la truie nullipare modifiait la composition du gain de poids durant cette période (appréciée selon le pourcentage des différents morceaux de découpe). Ainsi, entre 30 et 105 jours de gestation, le gain de poids correspondant à la longe, donc représentatif des masses musculaires, relativement à la bardière, représentatif de la masse adipeuse, peut varier du simple au double quand on augmente le taux protéique du régime de gestation.

Cette estimation de la composition corporelle, par la découpe de la carcasse, reste cependant imparfaite et il est donc nécessaire d'y associer d'autres paramètres permettant une approche plus précise : c'est le but de cette expérience dont l'objectif est triple : apprécier d'abord les modifications du métabolisme maternel et de la composition corporelle après une gestation en fonction de l'apport protéique ; caractériser ensuite la nature de la perte de poids au cours de la lactation; déterminer enfin la composition corporelle des animaux après le sevrage de la portée.

\section{Matériel et méthodes}

L'expérience a été entreprise sur 58 truies de race Large White, d'âge et de poids moyens à la puberté, respectivement de 239 jours et de $119 \mathrm{~kg}$. Durant la gestation (ou pendant une période équivalente pour les truies non gravides), les animaux sont répartis en deux lots ne différant que par l'apport protéique : selon un même niveau de rationnement de $2 \mathrm{~kg}$ par jour, soit $5900 \mathrm{Kcal}$ d'énergie digestible (ETIENNE, non publié), l'un des deux groupes reçoit un régime à 13,5 p. 100 de protéines (orgetourteau de soja, noté NP), l'autre groupe, un régime à base d'orge (noté BP) renfermant 10 p. 100 de protéines (composition des régimes : tabl. 1). En lactation, les animaux reçoivent le même régime NP selon un niveau alimentaire moyen de $5,1 \mathrm{~kg}$ par jour. Cet aliment est également distribué après le sevrage à raison de $1 \mathrm{~kg}$ (premier jour) puis $2 \mathrm{~kg}$ par jour.

Dans chaque lot, les truies sont affectées à l'une des quatre situations suivantes : truies non gravides recevant le régime expérimental au même niveau de consomma- 
tion et abattues 114 jours après la puberté (NG); truies saillies à la puberté et abattues dans les 48 heures après la parturition (PP), au $21^{\circ}$ jour de lactation (L) ou 8 jours après le sevrage ( $\mathrm{S}$; durée de la lactation : 35 jours).

\section{TABLEAU 1}

Composition des régimes.

Composition of experimental diets.

\begin{tabular}{|c|c|c|}
\hline $\begin{array}{c}\text { LOT } \\
\text { Source protéique } \\
\text { Protein source }\end{array}$ & $\begin{array}{c}\text { BP } \\
\text { Orge } \\
\text { Barley }\end{array}$ & $\begin{array}{c}\text { NP } \\
\text { Orge-soja } \\
\text { Barley-soyabean meal }\end{array}$ \\
\hline \multicolumn{3}{|l|}{ Composition (p. 100)) } \\
\hline$\underset{\text { Barley }}{\text { Orge }} \ldots \ldots \ldots \ldots \ldots \ldots \ldots \ldots \ldots \ldots \ldots \ldots \ldots$ & 96 & 86 \\
\hline $\begin{array}{l}\text { Tourteau de soja } \ldots \ldots \ldots \ldots \ldots \ldots \ldots \\
\text { Soyabean meal }\end{array}$ & - & 10 \\
\hline $\begin{array}{l}\text { Mélange minéral (1) } \ldots \ldots \ldots \ldots \ldots \ldots \ldots \\
\text { Mineral mixture }\end{array}$ & 3,6 & 3,6 \\
\hline $\begin{array}{l}\text { Mélange vitaminique (2) } \ldots \ldots \ldots \ldots \ldots \ldots \\
\text { Vitamin mixture }\end{array}$ & 0,4 & 0,4 \\
\hline \multicolumn{3}{|l|}{$\begin{array}{c}\text { Résultats d'analyses (p. 100) } \\
\text { Analytical results }\end{array}$} \\
\hline $\begin{array}{l}\text { Matière sèche } \ldots \ldots \ldots \ldots \ldots \ldots \ldots \\
\text { Dry matter }\end{array}$ & 87,3 & 87,8 \\
\hline $\begin{array}{l}\text { Matières azotées }(\mathrm{N} \times 6,25) \ldots \ldots \ldots \ldots \ldots \\
\text { Crude protein }\end{array}$ & 9,9 & 13,5 \\
\hline $\begin{array}{l}\text { Energie brute }(\mathrm{Kcal} / \mathrm{kg}) \ldots \ldots \ldots \ldots \ldots \ldots \\
\text { Gross energy }\end{array}$ & 3702 & 3742 \\
\hline
\end{tabular}

(1) Composition du mélange minéral (en p. 100 du régime) - Composition of mineral mixture (p. 100 of the diet) :

Phosphate bicalcique : 2,52 ; Carbonate de calcium : 0,67 ; Chlorure de sodium : 0,36; Oligoéléments : 0,05 .

(2) Composition du mélange vitaminique (en p. 100 du mélange) - Composition of vitamin mixture (p. 100 of the mixture) :

Vitamine $D_{3}(100000): 0,25$; Riboflavine : 0,1 ; Acide ascorbique : 0,1 ; Nicotinamide : 0,2; Pantothénate de Calcium : 0,25; Vitamine A $(50000): 2,0 ;$ Vitamine $B_{12}(100 \mathrm{mg} / \mathrm{kg}): 2,0$; Choline (25 p. 100): 10,0 Glucose anhydre qsp. 
Durant cette étude, les variations pondérales des truies sont enregistrées ainsi que leurs performances de reproduction à la mise bas et à 21 jours. Des mesures de production laitière sont faites par pesée des porcelets avant et après têtée, tous les cinq jours, selon une technique décrite par Salmon-Legagneur (1956). Le lendemain, un échantillon représentatif d'une têtée est prélevé afin d'en déterminer la composition globale (matière sèche, cendres, azote, lactose, énergie).

Vers le $80^{\circ}$ jour de gestation, l'ensemble des animaux est soumis, après une semaine d'adaptation, à une période de 5 jours en cage de digestibilité pendant laquelle on récolte séparément les fèces et l'urine dans le but de déterminer la digestibilité apparente des matières azotées ingérées et la rétention d'azote. Ces mesures sont renouvelées du $11^{\mathrm{e}}$ au $20^{\circ}$ jour de lactation, pour 6 animaux par lot (double période de collectes de 5 jours chacune par truie) et durant la première semaine après le sevrage, pour 7 animaux par lot.

A trois stades de la période expérimentale (30 et 105 jours de gestation, 10 jours de lactation), des prélèvements de sang sont effectués au niveau de la veine cave antérieure, après un jeûne d'une nuit. Sur ces échantillons sont réalisés un dosage (colorimétrique) de l'hémoglobine et de l'urée ainsi que la détermination des teneurs en acides aminés libres selon une technique décrite par DueE \& RERAT (1975).

Certains aspects du métabolisme du tissu adipeux (activité de la lipoprotéine lipase, synthèse de novo de lipides, mesurée in vitro) ont été également appréciés. Pour ce faire, des fragments de 4 à $5 \mathrm{~g}$ de lard sont prélevés au niveau du cou des truies, à 3 stades de gestation ( $30,60,105$ jours) et à 10 jours de lactation. Ces prélèvements sont obtenus sous anesthésie générale au thiopental sodique, après une nuit de jeûne. Le même type de prélèvement est effectué au moment de l'abattage des animaux. La cellularité du tissu adipeux est mesurée par la technique de RoDBell (1964). Les fragments de tissu sont dilacérés et placés pendant une heure à $37^{\circ} \mathrm{C}$ dans un milieu KREBS-RINGER, additionné de sérum-albumine bovine, auquel on a ajouté de la collagénase. Après rinçage de la solution, les adipocytes sont classés en fonction de leur diamètre à l'aide d'un micromètre après microphotographie. L'activité de la lipoprotéine lipase est mesurée après homogénéisation dans un tampon ammoniacal hépariné, selon une méthode décrite par PASCAL et al. (1977); les résultats sont exprimés en $\mu$ eq d'acides gras libérés (acide palmitique) par heure et par $\mathrm{g}$ de tissu dans le milieu d'incubation. Enfin, l'intensité de la lipogénèse dans le tissu adipeux est appréciée par la méthode de O'Hea \& Leveille (1968). Des coupes de tissu d'une centaine de mg sont mises à incuber, à $37^{\circ} \mathrm{C}$ pendant 2 heures, dans une solution de Krebs-Ringer sous atmosphère $\mathrm{O}_{2} \cdot \mathrm{CO}_{2}(19 / 1)$ avec agitation et en présence de glucose $\mathrm{U}{ }^{14} \mathrm{C}$. Après l'incubation, on mesure la radioactivité du gaz carbonique formé, des acides gras et du glycérol séparés après extraction des lipides et saponification.

A l'abattage des animaux, afin d'apprécier leur composition corporelle, différentes mesures sont effectuées à chaud ou après un ressuyage de 24 heures en chambre froide :

- pesée des carcasses avec tête et sans glande mammaire; mesures sur la carcasse chaude de l'épaisseur de lard sur la fente médiane au niveau du cou ( $7^{\mathbf{e}}$ vertèbre cervicale - $1^{\text {re }}$ dorsale), au niveau du dos $\left(13^{*}, 14^{\text {* }}\right.$ vertèbre dorsale), au niveau du rein ( $6^{\mathrm{e}}$ vertèbre lombaire). Mesure latérale de l'épaisseur de lard, à $6,5 \mathrm{~cm}$ de la fente, au niveau des $13^{\circ}, 14^{\circ}$ vertèbres dorsales ;

- découpe parisienne de la demi-carcasse, après un ressuyage de $24 \mathrm{~h}$; poids et densité du jambon et du « rein»; 
- sur la section transversale du rein, mesure de la surface du muscle long dorsal, selon la technique des points.

L'étude statistique des différents paramètres est réalisée au moyen du test d'analyse de variance.

\section{Résultats}

A. Variations pondérales des truies et performances de reproduction

L'administration du régime à 13,5 p. 100 de matières azotées durant la gestation permet aux jeunes truies un gain de poids supplémentaire d'environ 16 p. 100 qui cor. respond essentiellement à un accroissement du gain net (23 p. 100), sans modification du poids de portée à la naissance (tabl. 2). En ce qui concerne les truies non gravides, le supplément de poids induit par l'accroissement de consommation en protéines est moindre : ainsi, le gain net après 114 jours de période expérimentale n'augmente que de 11 p. 100.

\section{TABLEAU 2}

Performances de reproduction à la mise bas.

Reproductive performance at parturition.

\begin{tabular}{|c|c|c|c|}
\hline $\begin{array}{l}\text { LOT } \\
\text { Source protéique } \\
\text { Protein source }\end{array}$ & $\begin{array}{c}\text { BP } \\
\text { Orge } \\
\text { Barley }\end{array}$ & $\begin{array}{c}\text { NP } \\
\text { Orge-soja } \\
\text { Barley-Soyabean } \\
\text { meal }\end{array}$ & $\begin{array}{c}\text { Signification } \\
\text { statistique } \\
\text { S̄̄ (C.V.) } \\
\text { Statistical } \\
\text { significance }\end{array}$ \\
\hline $\begin{array}{l}\text { Gain brut de gestation }(\mathrm{kg}) \ldots \ldots \ldots \ldots \\
\text { Total weight gain during gestation }\end{array}$ & 41,7 & 48,3 & $1,9(18,9)(*)$ \\
\hline $\begin{array}{l}\text { Gain net }(\mathrm{kg}) \ldots \ldots \ldots \ldots \ldots \ldots \ldots \\
\text { Net weight gain }\end{array}$ & & & \\
\hline - truies gravides $(2) \ldots \ldots \ldots \ldots \ldots \ldots$ & 26,9 & 33,0 & $1,9(28,9)(*)$ \\
\hline $\begin{array}{l}\text { - truies non gravides (3) } \ldots \ldots \ldots \ldots \text {. } \\
\text { non pregnant gilts }\end{array}$ & 25,9 & 28,9 & $2,5(26,4) \mathrm{NS}$ \\
\hline $\begin{array}{l}\text { Nombre total de porcelets } \ldots \ldots \ldots \ldots \\
\text { Total number of piglets }\end{array}$ & 8,5 & 9,2 & $0,6(32,6) \mathrm{NS}$ \\
\hline $\begin{array}{l}\text { Nombre de porcelets nés vivants } \ldots \ldots \\
\text { Number of piglets born alive }\end{array}$ & 8,2 & 8,7 & $0,6(34,1) \mathrm{NS}$ \\
\hline $\begin{array}{l}\text { Poids moyen des porcelets }(\mathrm{kg}) \ldots \ldots \ldots \\
\text { Mean weight of piglets }\end{array}$ & 1,2 & 1,15 & $0,05(15,8) \mathrm{NS}$ \\
\hline
\end{tabular}

(1) Ecart-type de la moyenne (coefficient de variation). NS : différence non significative - Standard error of the mean (coefficient of variation). NS: non significant.

$(*)$ Différence significatıve au seuil $\mathrm{p}<0,05 ;(* *): \mathrm{p}<0,01 ;(* * *): \mathrm{p}<0,001-$ Levels of significance : $(*): 0.05 ;(* *): 0.01 ;(* * *): 0.001$.

(2) Nombre de truies gravides par lot : 21 - Number of pregnant gilts per lot : 21 .

(3) Nombre de truies non gravides par lot : 8 - Number of non pregnant gilts per lot : 8 . 
TABLEAU 3

Performances au cours de la lactation (0-21 jours).

Performance during lactation (0-21 days).

\begin{tabular}{|c|c|c|c|c|}
\hline $\begin{array}{l}\text { LOT } \\
\text { Source protéique en gestation } \\
\text { Protein source in gestation diet }\end{array}$ & $\begin{array}{l}\text { BP } \\
\text { Orge } \\
\text { Barley }\end{array}$ & $\begin{array}{c}\text { NP } \\
\text { Orge-Soja } \\
\begin{array}{c}\text { Barley-Soyabean } \\
\text { meal }\end{array}\end{array}$ & $\begin{array}{c}\text { Signification } \\
\text { statistique } \\
\mathrm{S} \overline{\mathrm{x}} \text { (C.V.) } \\
\text { Statistical } \\
\text { significance }\end{array}$ & \\
\hline $\begin{array}{l}\left.\text { Nombre de truies }{ }^{2}\right) \ldots \ldots \ldots \ldots \ldots \ldots \\
\text { Number of sows }\end{array}$ & 14 & 14 & & \\
\hline $\begin{array}{l}\text { Variation de poids }(\mathrm{kg}) \ldots \ldots \ldots \ldots \ldots \\
\text { Live-weight variation }\end{array}$ & $-8,5$ & $-6,8$ & $3,1(151,1)$ & NS \\
\hline $\begin{array}{l}\text { Nombre de porcelets à } 21 \text { jours ( } 3 \text { ) } \\
\text { Number of piglets at } 21 \text { days }\end{array}$ & 8,5 & 8,0 & $0,6 \quad(25,3)$ & NS \\
\hline $\begin{array}{l}\text { Gain de poids de la portée }(\mathrm{kg}) \ldots \ldots \\
\text { Litter gain }\end{array}$ & 23,5 & 22,5 & $1,8 \quad(28,8)$ & NS \\
\hline $\begin{array}{l}\text { Production laitière }(\mathrm{kg} / \mathrm{j})^{(4)} \\
\text { Milk production }(\mathrm{kg} / \mathrm{d})\end{array}$ & 5,15 & 4,9 & $0,4 \quad(24,0)$ & NS \\
\hline
\end{tabular}

(1) Voir tableau 2 - See table 2.

(2) Consommation moyenne : $5,1 \mathrm{~kg}$ par jour - Mean feed intake : $5.1 \mathrm{~kg}$ per day.

(3) Nombre moyen de porcelets à la naissance : 9,4 - Mean number of piglets at birth : 9.4.

(4) Estimée à partir de 4 déterminations par truie et 8 truies par lot - Estimated after foul determinations per sow and eight sows per group.

En lactation, l'étude porte principalement sur les trois premières semaines (tabl. 3) et sur un effectif d'animaux plus faible. On constate que, l'apport de protéines étant identique durant cette période (régime NP distribué à l'ensemble des animaux), la perte de poids est comparable quel qu'ait été le régime de gestation. De la même façon, quand on considère la croissance de la portée (taille de la portée $\times$ croissance moyenne du porcelet entre 0 et 21 jours) ou la production laitière, il n'apparaît pas de différence selon l'apport protéique distribué en gestation. Par ailleurs, la composition du lait de truie ne semble pas être influencée par le régime nutritionnel de gestation.

Après le sevrage, compte tenu des abattages intermédiaires, les observations ne portent que sur 7 animaux par lot dont les performances au cours de la lactation ne reflètent qu'imparfaitement les valeurs moyennes établies précédemment (taille de portée et production laitière plus faibles); néanmoins, ces animaux subissent une perte de poids moyenne d'environ $2 \mathrm{~kg}$ pendant la première semaine après le sevrage. 


\section{B. Utilisation digestive et métabolique de l'azote ingéré}

L'administration du régime à 13,5 p. 100 de matières azotées à la truie gravide comme à la truie non gravide ne semble pas modifier l'excrétion azotée fécale (tabl. 4). Il s'ensuit que l'utilisation digestive apparente de l'azote ingéré, qui ne tient pas compte des pertes endogènes, augmente au taux protéique le plus élevé. Par contre, l'excrétion azotée urinaire est accrue, principalement chez les animaux non gravides. En conséquence, le bilan azoté des femelles non saillies reste invariant lorsqu'elles augmentent leur consommation protéique. Chez les truies gravides, par contre, le bilan azoté est amélioré (d'environ 30 p. 100). Toutefois, pour un même apport protéique, les différences selon l'état physiologique sont minimes.

\section{TABLEAU 4}

Utilisation digestive et métabolique de l'azote ingéré en gestation.

Digestible and metabolic utilization of nitrogen ingested during gestation.

\begin{tabular}{|c|c|c|c|c|c|}
\hline \multirow[t]{2}{*}{$\begin{array}{l}\text { LOT } \\
\text { Source protéique } \\
\text { Protein source }\end{array}$} & \multicolumn{2}{|c|}{$\begin{array}{c}\text { BP } \\
\text { Orge } \\
\text { Barley }\end{array}$} & \multicolumn{2}{|c|}{$\begin{array}{c}\text { NP } \\
\text { Orge-Soja } \\
\text { Barley-Soyabean } \\
\text { meal }\end{array}$} & \multirow{2}{*}{$\begin{array}{c}\text { Sx }(C . V .)(1) \\
\text { Signification } \\
\text { statistique } \\
\text { Statistical } \\
\text { significance }\end{array}$} \\
\hline & $G(2)$ & NG & G & $\mathrm{NG}$ & \\
\hline $\begin{array}{l}\text { Azote ingéré }(\mathrm{g} / \text { jour }) \ldots \ldots \ldots \ldots \\
\text { Nitrogen ingested }(\mathrm{g} / \text { day })\end{array}$ & 33,3 & 33,1 & 43,4 & 43,4 & \\
\hline $\begin{array}{l}\text { Azote fécal ( } \mathrm{g} / \text { jour }) \\
\text { Fecal nitrogen }(\mathrm{g} / \text { day })\end{array}$ & 8,0 & 7,9 & 8,6 & 7,8 & $0,3(16,9) \mathrm{NS}$ \\
\hline $\begin{array}{l}\text { Utilisation digestive apparente de } \\
\text { l'azote }(\%) \ldots \ldots \ldots \ldots \ldots \ldots \ldots \ldots \\
\text { Apparent digestibility of nitrogen }\end{array}$ & 76,0 & 75,8 & 80,0 & 82,0 & $0,8 \quad(5,0)(* *)$ \\
\hline $\begin{array}{l}\text { Azote urinaire }(g / \text { jour }) \\
\text { Urinary nitrogen }(g / \text { day })\end{array}$ & 16,0 & 15,2 & 22,8 & 25,2 & $1,2 \cdot(28,0)(* * *)$ \\
\hline $\begin{array}{l}\text { Azote reteru }(g / \text { jour }) \ldots \ldots \ldots \ldots \\
\text { Nitrogen retention }(g / \text { day })\end{array}$ & 9,3 & 10,0 & 12,0 & 10,4 & $1,2(51,9) \mathrm{NS}$ \\
\hline $\begin{array}{l}\text { Coefficient de rétention de l'azo- } \\
\text { te }(\%) \ldots \ldots \ldots \ldots \ldots \ldots \ldots \ldots \ldots \\
\text { Nitrogen retention coefficient }\end{array}$ & 36,7 & 39,0 & 34,5 & 29,1 & $3,6(46,3)$ NS \\
\hline
\end{tabular}

(1) Voir tableau 2 - See table 2

(2) $\mathrm{G}=$ truies gravides - pregnant gilts; $\mathrm{NG}=$ truies non gravides - non pregnant gilts.

En lactation, la consommation azotée moyenne, par rapport à la période précédente est nettement accrue (de 2,4 à 3,2 fois plus). L'excrétion azotée fécale est également augmentée, dans des proportions comparables (tabl. 5). L'utilisation digestive apparente de l'azote reste donc similaire d'un stade physiologique à l'autre. 


\section{Tableau 5}

Utilisation digestive et métabolique de l'azote ingéré en lactation (1).

Digestible and metabolic utilization of nitrogen ingested during lactation.

\begin{tabular}{|c|c|c|c|}
\hline $\begin{array}{l}\text { LOT } \\
\text { Source protéique en gestation } \\
\text { Protein source in gestation diet }\end{array}$ & $\begin{array}{c}\text { BP } \\
\text { Orge } \\
\text { Barley }\end{array}$ & \begin{tabular}{|} 
NP \\
Orge-Soja \\
$\begin{array}{c}\text { Barley-Soyabean } \\
\text { meal }\end{array}$
\end{tabular} & $\begin{array}{c}\text { Signification } \\
\text { statistique } \\
\text { S̄̃ (C.V.) } \\
\text { Statistical } \\
\text { significance }\end{array}$ \\
\hline $\begin{array}{l}\text { Azote ingéré ( } \mathrm{g} / \text { jour }) \\
\text { Nitrogen ingested }(\mathrm{g} / \text { day })\end{array}$ & 105,8 & 104,5 & \\
\hline $\begin{array}{l}\text { Azote fécal }(\mathrm{g} / \text { jour }) \ldots \ldots \ldots \ldots \ldots \ldots \\
\text { Fecal nitrogen }(g / \text { day })\end{array}$ & 20,0 & 18,15 & $1,3 \quad(23,9)$ NS \\
\hline $\begin{array}{l}\text { Utilisation digestive apparente de l'azo- } \\
\text { te }(\%) \ldots \ldots \ldots \ldots \ldots \ldots \ldots \ldots \ldots \ldots \ldots \\
\text { Apparent digestibility of nitrogen }\end{array}$ & 81,3 & 82,5 & $1,2 \quad(5,0) \mathrm{NS}$ \\
\hline $\begin{array}{l}\text { Azote urinaire }(\mathrm{g} / \mathrm{jour}) \\
\text { Urinary nitrogen }(\mathrm{g} / \text { day })\end{array}$ & 24,4 & 23,05 & $1,9 \quad(27,7) \mathrm{NS}$ \\
\hline $\begin{array}{l}\text { Azote du iait }(\mathrm{g} / \text { jour }) \ldots \ldots \ldots \ldots \ldots \ldots \\
\text { Nitrogen in milk }(g / \text { day })\end{array}$ & 56,1 & 55,7 & $3,9 \quad(24,3) \mathrm{NS}$ \\
\hline $\begin{array}{l}\text { Azote retenu }(\mathrm{g} / \text { jour }) \ldots \ldots \ldots \ldots \ldots \ldots \\
\text { Nitrogen retention }(\mathrm{g} / \text { day })\end{array}$ & 5,3 & 7,6 & $3,7(200,0) \mathrm{NS}$ \\
\hline
\end{tabular}

(1) $\mathrm{Du} 11^{\mathrm{C}}$ au $20^{\mathrm{e}}$ jour de lactation, après double mesure sur six animaux par lot. Après 3 semaines de lactation, le nombre de porcelets allaités et la perte de poids sont respectivement de 9,1 et 11,3 kg (lot 1);8,9 et 12,2 $\mathrm{kg}$ ( $(\mathrm{lot} 2)$ - Between day 11 and day 20 of lactation, after two measures on six animals per group. After three weeks of lactation, the number of suckled piglets per sow and the liveweight loss were, respectively: 9.1 and $11.3 \mathrm{~kg}$ (lot 1$) ; 8.9$ and $12.2 \mathrm{~kg}$ (lot 2).

(2) Voir tableau 2 - See table 2.

Après absorption, l'utilisation de l'azote est représentée principalement par la synthèse et l'exportation des protéines dans le lait ( $356 \mathrm{~g}$ par jour en moyenne à ce stade). On constate que l'excrétion azotée urinaire demeure modeste. Le bilan azoté global, faibiement positif, ne semble pas alors affecté par lapport protéique durant la gestation précédente. Il en est de même après le sevrage où le bilan azoté des animaux est comparable à celui établi à 80 jours de gestation (résultats non présentés).

\section{Variations des paramètres sanguins}

Leétude des concentrations en hémoglobine du sang (résultats non présentés) ne montre pas d'effet de l'apport protéique au cours de la gestation. Par contre, si, au bout de 30 jours d'étude, la valeur obtenue est identique chez les truies gravides 
ou non $(16,4 \pm 0,4 \mathrm{~g} / 100 \mathrm{ml})$, il existe en fin de gestation une différence significative entre les deux groupes : la concentration en hémoglobine diminuant chez les truies gravides $(14,1 \pm 0,3$ contre $16,0 \pm 0,5 \mathrm{~g} / 100 \mathrm{ml})$. En lactation, la teneur en hémoglobine augmente $(15,55 \pm 0,60 \mathrm{~g} / 100 \mathrm{ml})$ retrouvant une valeur proche de la valeur initiale.

L'étude des concentrations en urée dans le sang total (résultats non présentés) fait apparaître, chez les truies non gravides, une tendance à l'augmentation (de 10 p. 100 environ) avec l'apport protéique le plus élevé (moyennes établies sur des valeurs collectées aux deux stades de la période d'étude). Chez les truies gravides, par contre, l'évolution de l'urémie au cours de la gestation diffère selon l'apport protéique. Si, à 10 p. 100 de protéines, la concentration en urée dans le sang s'élève de 10 p. 100 en fin de gestation, l'évolution inverse est observée au taux protéique le plus élevé. En lactation, enfin, l'urémie est accrue chez tous les animaux.

En ce qui concerne les teneurs en acides aminés libres du sang, obtenues après une courte période de jeûne, en fin de gestation, l'ampleur des variations est modeste (tabl. 6). On remarquera qu'au taux protéique le plus élevé, les différences entre animaux gravides ou non portent essentiellement sur certains acides aminés non indispensables (acide aspartique, alanine, glycine, glutamine) dont les teneurs sont plus élevées en gestation. Parallèlement, dans le groupe des acides aminés indispensables, seule la concentration en valine a tendance à diminuer. A 10 p. 100 de protéines dans la ration, laugmentation, déjà constatée chez les truies gravides, du groupe des acides aminés non indispensables (Asp, Ala, Gly, Gln) coexiste avec une diminution des teneurs en acides aminés ramifiés (Val, Leu) et en lysine.

En lactation enfin, les animaux consommant le même régime NP, on note, par rapport aux truies non gravides du lot NP, une augmentation en certains acides aminés non indispensables (Asp, Glu, Gln, Ala), associée à une diminution des teneurs en quelques acides aminés indispensables (Leu, Val, Lys).

Indépendamment de l'état physiologique, l'augmentation du taux protéique dans la ration provoque une augmentation de la teneur en lysine d'une part et une chute de certains acides aminés non indispensables (Pro, Glu, et secondairement Asp et Ser) ou indispensables (Thr, Phe) d'autre part.

\section{Métabolisme du tissu adipeux sous-cutané}

La taille des adipocytes du tissu adipeux sous-cutané, au niveau du cou, présente une faible amplitude de variations (tabl. 7). Cependant, si l'on considère que le volume de l'adipocyte est proportionnel au cube de son rayon, les différences entre stades peuvent être accentuées. Il en est ainsi de l'évolution correspondante chez les truies non gravides : une augmentation importante (en volume : 25 p. 100 environ) est notée au cours de la période d'étude; la taille des adipocytes étant systématiquement plus faible chez les animaux non gravides recevant l'apport protéique le plus bas. Par contre, ce type d'évolution n'est plus constaté chez les truies gravides. Si la taille des adipocytes reste constante au niveau d'apport protéique le plus bas, l'évolution est biphasique au niveau protéique le plus élevé (augmentation jusqu’à 60 jours de gestation puis diminution en fin de gestation). Enfin, les données collectées au cours de la lactation mettent en évidence une augmentation du diamètre moyen des adipocytes quel que soit l'apport protéique durant la gestation. Cette valeur plus élevée est confirmée après le sevrage $(89.4 \mu)$, sur peu d'animaux, il est vrai (données non présentées). 


\section{TABLEAU 6}

Variations de l'aminoacidémie libre en fonction de l'apport protéique et de l'état physiologique (en $\mathrm{mg}$ p. $100 \mathrm{~g}$ de sang).

Free amino acids in blood according to protein intake and physiological status (in $\mathrm{mg} \mathrm{p.} 100 \mathrm{~g}$ blood).

\begin{tabular}{|c|c|c|c|c|c|}
\hline Taux protéique $(\%)$ & \multicolumn{2}{|c|}{$10(\mathrm{BP})$} & \multicolumn{2}{|c|}{$13,5(\mathrm{NP})$} & $13,5(\mathrm{NP})$ \\
\hline $\begin{array}{c}\text { Etat physiologique (1) } \\
\text { Physiological status }\end{array}$ & NG & G & NG & G & $\mathrm{L}$ \\
\hline $\begin{array}{c}\text { Nombre de données (2) } \\
\text { Number } \\
\text { of determinations }\end{array}$ & 7 & 11 & 7 & 11 & 18 \\
\hline Asp .. & $0,7 \pm 0,1$ & $1,05 \pm 0,1$ & $0,55 \pm 0,05$ & $0,8 \pm 0,15$ & $0,9 \pm 0,1$ \\
\hline Thr & $1,65 \pm 0,1$ & $1,7 \pm 0,1$ & $1,5 \pm 0,1$ & $1,45 \pm 0,05$ & $1,4 \pm 0,05$ \\
\hline Ser & $1,5 \pm 0,15$ & $1,45 \pm 0,05$ & $1,3 \pm 0,05$ & $1,3 \pm 0,05$ & $1,3 \pm 0,05$ \\
\hline Glu & $2,8 \pm 0,3$ & $2,65 \pm 0,15$ & $2,35 \pm 0,25$ & $2,2 \pm 0,25$ & $2,8 \pm 0,15$ \\
\hline Gin & $1,6 \pm 0,1$ & $1,85 \pm 0,15$ & $1,25 \pm 0,15$ & $2,1 \pm 0,2$ & $3,1 \pm 0,2$ \\
\hline Pro & $4,85 \pm 0,5$ & $4,8 \pm 0,4$ & $4,05 \pm 0,2$ & $3,75 \pm 0,15$ & $4,15 \pm 0,2$ \\
\hline Gly & $5,45 \pm 0,5$ & $5,95 \pm 0,5$ & $5,6 \pm 0,35$ & $5,95 \pm 0,2$ & $5,55 \pm 0,2$ \\
\hline Ala & $4,15 \pm 0,5$ & $4,65 \pm 0,3$ & $3,4 \pm 0,15$ & $4,2 \pm 0,3$ & $4,5 \pm 0,2$ \\
\hline Cit ... & $1,85 \pm 0,3$ & $1,45 \pm 0,1$ & $1,4 \pm 0,1$ & $1,2 \pm 0,05$ & $0,95 \pm 0,05$ \\
\hline Val & $4,05 \pm 0,15$ & $3,25 \pm 0,15$ & $3,6 \pm 0,2$ & $3,15 \pm 0,2$ & $3,25 \pm 0,1$ \\
\hline Cys & $0,4 \pm 0,05$ & $0,45 \pm 0,05$ & $0,4 \pm 0,05$ & $0,5 \pm 0,05$ & $0,5 \pm 0,05$ \\
\hline Met & $0,45 \pm 0,05$ & $0,5 \pm 0,05$ & $0,4 \pm 0,05$ & $0,45 \pm 0,01$ & $0,45 \pm 0,02$ \\
\hline Ile & $1,55 \pm 0,05$ & $1,55 \pm 0,05$ & $1,3 \pm 0,05$ & $1,7 \pm 0,15$ & $1,50 \pm 0,05$ \\
\hline Leu & $2,45 \pm 0,1$ & $2,1 \pm 0,05$ & $2,3 \pm 0,1$ & $2,35 \pm 0,2$ & $2,0 \pm 0,1$ \\
\hline Tyr & $1,5 \pm 0,1$ & $1,4 \pm 0,05$ & $1,35 \pm 0,05$ & $1,45 \pm 0,05$ & $1,65 \pm 0,05$ \\
\hline Phe & $1,95 \pm 0,1$ & $1,9 \pm 0,1$ & $1,55 \pm 0,1$ & $1,65 \pm 0,05$ & $1,5 \pm 0,05$ \\
\hline Orn & $1,75 \pm 0,1$ & $1,55 \pm 0,05$ & $1,3 \pm 0,1$ & $1,5 \pm 0,1$ & $1,2 \pm 0,05$ \\
\hline Lys $\ldots \ldots \ldots \ldots$ & $2,0 \pm 0,2$ & $1,7 \pm 0,1$ & $2,3 \pm 0,1$ & $2,3 \pm 0,1$ & $1,95 \pm 0,1$ \\
\hline His & $1,3 \pm 0,05$ & $1,2 \pm 0,05$ & $1,2 \pm 0,05$ & $1,2 \pm 0,05$ & $1,3 \pm 0,05$ \\
\hline Arg & $0,9 \pm 0,1$ & $0,9 \pm 0,1$ & $0,95 \pm 0,1$ & $0,95 \pm 0,1$ & $0,75 \pm 0,05$ \\
\hline
\end{tabular}

(1) $\mathrm{G}=$ truies gravides - pregnant gilts; $\mathrm{NG}=$ truies non gravides - non pregnant gilts; $\mathbf{L}=$ truies en lactation - lactating sows.

(2) Moyenne suivie de l'écart type de la moyenne - Mean value followed by standard deviation of the mean. 


\section{TABLeau 7}

Evolution de la taille des adipocytes au cours du cycle de reproduction, en fonction de l'apport protéique de gestation (en $\mu$ ).

Evolution of the adipocyte size during the reproductive cycle according to protein level in gestation diet (in $\mu$ ).

\begin{tabular}{|c|c|c|c|c|}
\hline \multirow{2}{*}{$\begin{array}{c}\text { Taux protéique en gestation }(\%) \\
\text { Protein level in gestation diet } \\
\text { Etat physiologique }(1) \\
\text { Physiological status }\end{array}$} & \multicolumn{2}{|c|}{$\begin{array}{l}10 \\
\mathrm{BP}\end{array}$} & \multicolumn{2}{|c|}{$\begin{array}{l}13,5 \\
\mathrm{NP}\end{array}$} \\
\hline & NG & G & NG & G \\
\hline - Gestation & & & & \\
\hline 30 j. (30 days) .. & 82,6 & 87,2 & 85,7 & $82 ; 2$ \\
\hline $60 \mathrm{j} .(60$ days $) \ldots \ldots \ldots$ & 85,2 & 87,4 & 90,1 & 87,5 \\
\hline parturition (2) $\quad \ldots \ldots \ldots$ & 89,6 & 86,5 & 91,5 & 84,5 \\
\hline - Lactation $(3)$ & & 91,6 & & 89,8 \\
\hline$S \bar{x}(C . V).(4)$ & $2,0(9,6)$ & $1,8(13,1)$ & $2,3(10,1)$ & $1,3(9,7)$ \\
\hline $\begin{array}{l}\text { Nombre de données .............. } \\
\text { Number of determinations }\end{array}$ & 17 & 40 & 15 & 43 \\
\hline
\end{tabular}

(1) Voir tableau 4 - See table 4.

(2) A 105 jours de gestation et 2 jours après parturition - On day 105 of gestation and 2 days after parturition.

(3) A 10 et 21 jours de lactation - On day 10 and 21 of lactation.

(4) Voir tableau 2 - See table 2.

L'activité de la lipoprotéine lipase du tissu adipeux, mesurée au niveau du cou, permet d'estimer la capacité de ce tissu à capter les triglycérides circulants. Dans la figure 1, sont regroupées les valeurs déterminées lorsque la biopsie de lard dorsal est effectuée sous anesthésie générale. Quoique la variabilité enregistrée à chaque stade soit très élevée, on constate cependant que l'évolution générale, au cours du cycle de reproduction, est comparable d'un lot à l'autre. Après une augmentation non significative à 60 jours de gestation, l'activité de la lipoprotéine lipase est diminuée en fin de gestation, cette diminution étant maintenue (lot BP) ou accentuée (lot NP) à 10 jours de lactation. Au cours de la gestation, les activités de la lipoprotéine lipase sont constamment plus élevées chez les animaux recevant le régime NP, les différences n'étant pas statistiquement significatives; une tendance contraire est à noter chez les animaux non gravides. Par ailleurs, les valeurs obtenues à l'abattage des animaux confirment une activité basse de la lipoprotéine lipase, dès le second jour après la parturition, sans effet du taux protéique de gestation $(11,4 \pm 2,0 \mu \mathrm{eq} / \mathrm{g} / \mathrm{h})$. Cette faible activité est maintenue à 21 jours de lactation $(8,9 \pm 1,9 \mu \mathrm{eq} / \mathrm{g} / \mathrm{h})$. Par contre, après le sevrage, une activité plus forte est constatée $(20,0 \pm 2,3 \mu \mathrm{eq} / \mathrm{g} / \mathrm{h})$.

L'intensité de la lipogénèse a été estimée par la transformation du glucose-U-14C en gaz carbonique marqué ou en lipides (acides gras et glycérol après séparation). Les valeurs reportées dans la figure 2 concernent les mesures obtenues sous anesthésie 
générale. On constate là encore une variabilité élevée à chaque stade. Il semble néanmoins que la production de gaz carbonique marqué, évolue de la même façon que la synthèse de lipides, quel que soit le stade physiologique ou le régime nutritionnel.

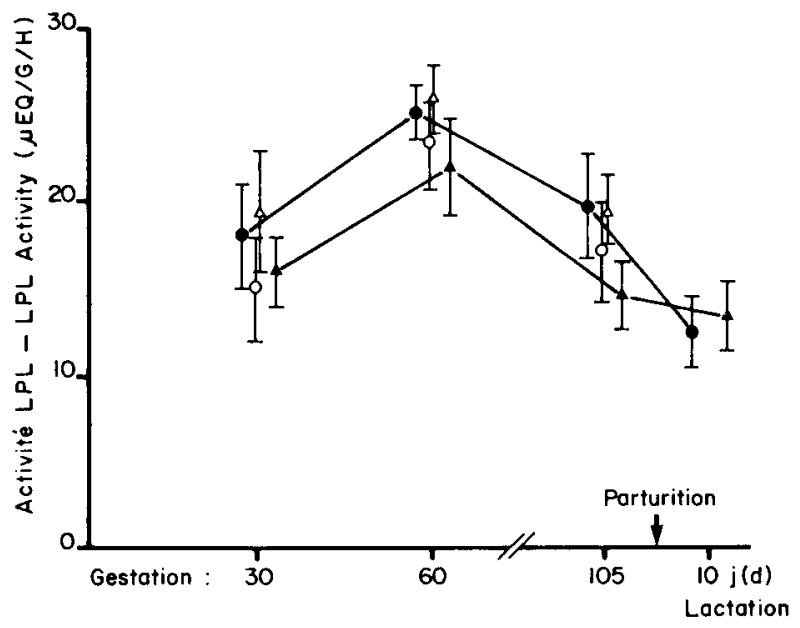

FIGURE 1

Activité de la lipoprotéine-lipase du tissu adipeux sous cutané $(\mu e q / \mathrm{g} / \mathrm{h})$, en fonction de l'état physiologique et du taux protéique de gestation.

Lipoprotein-lipase activity of backfat $(\mu e q / g / h)$, according to physiological status and protein level in gestation diet.

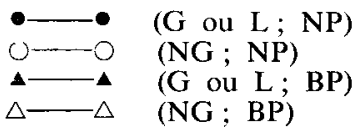

Nombre de déterminations par valeur moyenne : 5 à 12 .

Number of determinations for each mean value : 5 to 12.

Chez les truies non gravides, l'intensité de la lipogénèse tend à diminuer au fur et à mesure de l'expérience. Ce ralentissement apparaît précocement pour les animaux recevant le régime NP, plus tardivement pour l'autre groupe.

Une remarque similaire peut être formulée pour les truies gravides : l'intensité de la lipogénèse décroît en fin de gestation. On remarquera cependant qu'à 2 mois de gestation, l'intensité de la lipogénèse est franchement différente dans les deux groupes (la différence est significative au seuil de 5 p. 100) : les truies recevant l'apport pro. téique le plus élevé (régime NP) ont une activité lipogénique plus forte. En lactation (à 10 jours), le tissu adipeux sous-cutané des truies est caractérisé également par une activité lipogénique faible que confirment les valeurs obtenues à l'abattage des animaux ( 2 ou 21 jours). Indépendamment du stade physiologique, on trouve une intensité de la lipogénèse plus faible chez les truies recevant le régime $\mathrm{BP}$, contrairement aux truies non gravides. 


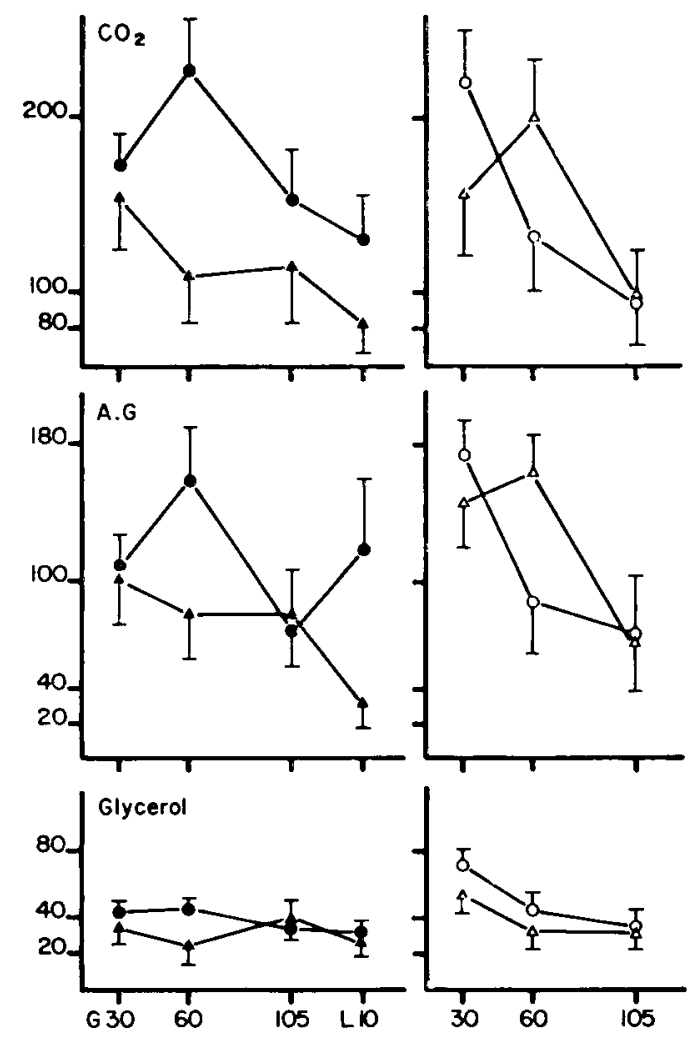

FIGURE 2

Activité métubolique du tissu adipeux sous-cutané mesurée par la production de $\mathrm{CO}_{2}$, d'acides gras $(A G)$ et de glycérol à partir de glucose- $U{ }^{14} \mathrm{C}$ (nanomoles de substrat transformél $100 \mathrm{mg} / 2 \mathrm{~h}$ ).

Backfat metabolic activity, measured by production of $\mathrm{CO}_{z}$, fatty acid and glycerol from glucose $-U-{ }^{14} \mathrm{C}$.

Abreviations et nombre de déterminations : voir figure 1 . Abbreviations and number of determinations : see figure 1 .

\section{E. Estimation de la composition corporelle à l'abattage}

La composition corporelle des animaux a été déterminée après prélèvement de certains compartiments (glande mammaire, tractus digestif ou reproducteur) dont l'ampleur est modifiée selon le stade physiologique d'abattage. Dans ces conditions, on peut considérer que le poids net de carcasse est comparable d'un stade physiologique à l'autre, excepté au stade «lactation » (à 21 jours) où le poids net de carcasse est alors significativement réduit (tabl. 8). Le rendement de carcasse y est également fortement déprécié.

Exprimés relativement au poids net (poids de la carcasse après un ressuyage de $24 \mathrm{~h}$ ), les poids des morceaux de découpe n'apparaissent pas significativement différents 


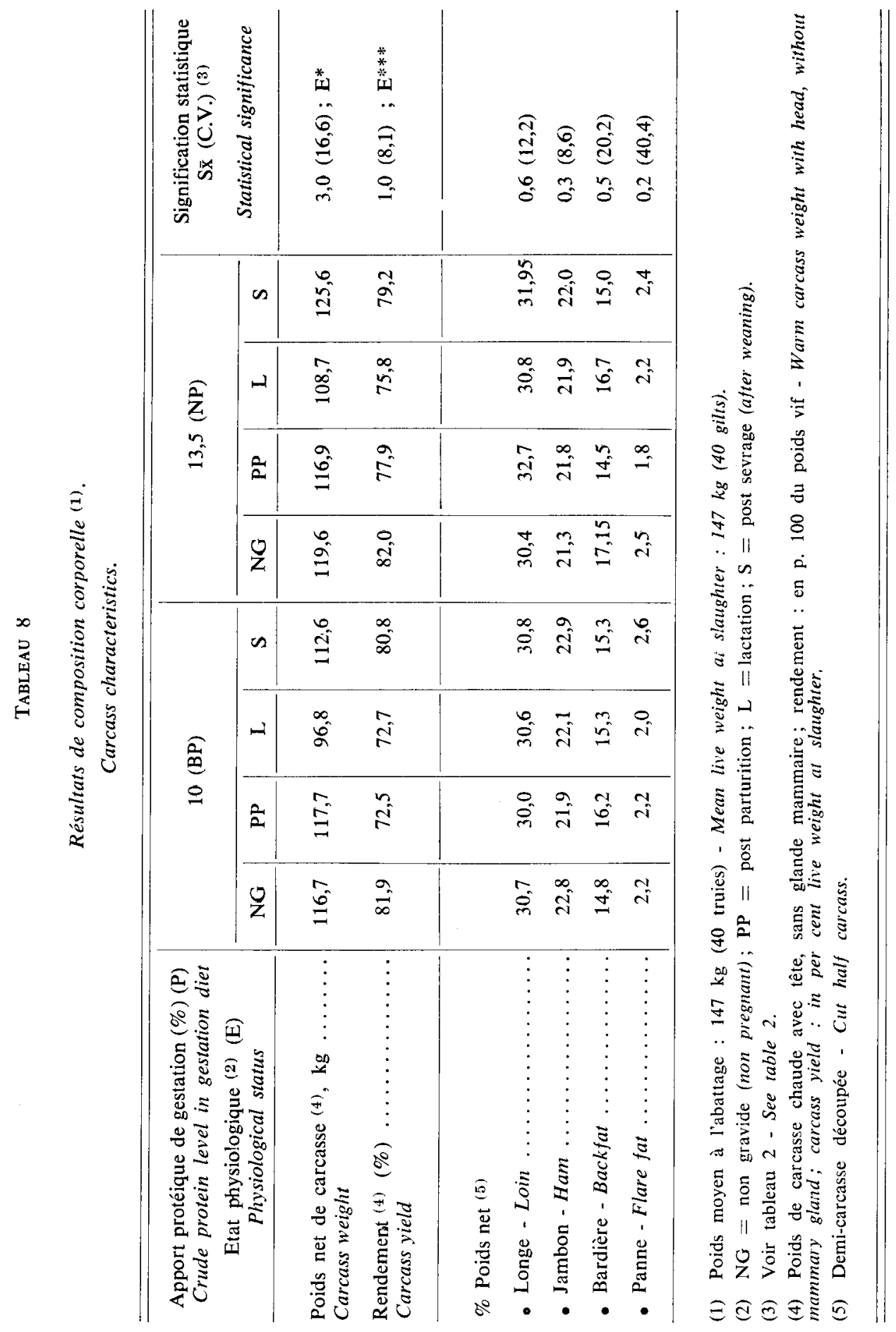


d'un groupe à l'autre. Ceci est particulièrement vrai pour le pourcentage de jambon. Toutefois, certaines tendances se dessinent : ainsi, chez les animaux non gravides, l'état d'engraissement est accru quand on augmente l'apport protéique; c'est la tendance inverse qui est observée pour les truies parturientes ou ayant terminé leur lactation; enfin, au pic de lactation, le poids relatif des différents morceaux est similaire quel que soit l'apport protéique durant la gestation précédente : en valeurs absolues par contre, ceci se traduit par une diminution de la quantité de longe dans la carcasse (dans les deux lots), ainsi que des masses adipeuses dans le lot correspondant à l'apport protéique le plus faible en gestation.

TABleau 9

Mesures déterminées après section du rein (1).

Determinations after section of back and loin.

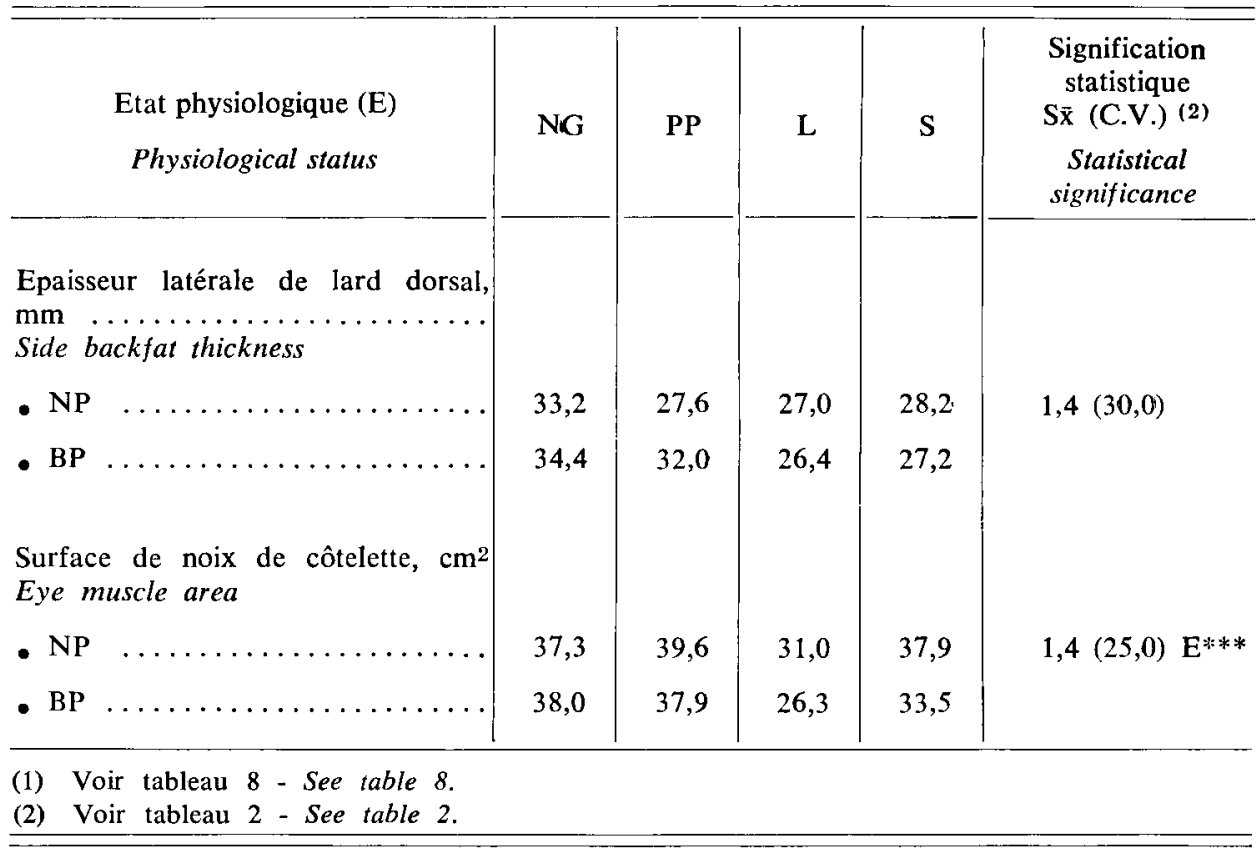

Les mesures d'épaisseur de lard dorsal à différents sites, perpendiculairement à la fente (résultats non présentés), montrent que la couverture graisseuse à la parturition est réduite quand l'animal reçoit l'apport protéique le plus élevé ; c'est l'évolution inverse qui est constatée chez la truie n'ayant pas accompli de gestation. En lactation, ou après le sevrage, les différences sont peu importantes : seule, une diminution de l'épaisseur de lard dorsal au niveau du dos à 21 jours de lactation (groupe BP) est à souligner.

L'épaisseur latérale de lard dorsal, au niveau des $13^{\circ}-14^{\circ}$ vertèbres dorsales, mesurée à $6,5 \mathrm{~cm}$ de la fente, illustre de la même manière les différences observées 
précédemment (tabl. 9) : l'état d'engraissement à la parturition, apprécié par ce biais, est réduit chez les truies ayant reçu le régime à 13,5 p. 100 de protéines en gestation, une mobilisation des graisses s'effectuant durant la lactation pour les truies de l'autre groupe. Inversement, la surface de noix de côtelette est légèrement accrue chez les parturientes qui ont reçu l'apport protéique le plus élevé (tabl. 9). En lactation, cependant, l'évolution de la surface de noix de côtelette est similaire : après une diminution très prononcée au pic de lactation, elle s'accroît après le sevrage, retrouvant une valeur plus ou moins voisine de la valeur initiale.

TABLEAU 10

Densité du rein et du jambon (1).

Back and loin and ham specific gravity.

\begin{tabular}{|c|c|c|c|c|c|}
\hline $\begin{array}{c}\text { Etat physiologique (E) } \\
\text { Physiological status }\end{array}$ & NG & PP & $\mathrm{L}$ & $\mathbf{S}$ & 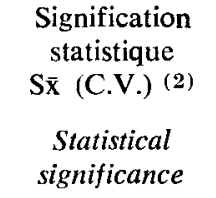 \\
\hline $\begin{array}{l}\text { Densité du rein } \\
\text { Back and loin specific gravity }\end{array}$ & & & & & \\
\hline 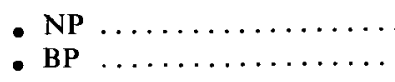 & $\begin{array}{l}1,031 \\
1,035\end{array}$ & $\begin{array}{l}1,047 \\
1,029\end{array}$ & $\begin{array}{l}1,033 \\
1,028\end{array}$ & $\begin{array}{l}1,040 \\
1,034\end{array}$ & $0,002(1,2) \mathrm{P} *$ \\
\hline $\begin{array}{l}\text { Densité du jambon } \\
\text { Ham specific gravity }\end{array}$ & & & & & \\
\hline - NP & 1,047 & 1,057 & 1,051 & 1,056 & $0,002(1,2) \quad P^{* *}$ \\
\hline - $\mathrm{BP}$ & 1,048 & 1,042 & 1,044 & 1,042 & \\
\hline
\end{tabular}

(1) Voir tableau 8 - See table 8 .

(2) Voir tableau 2 - See table 2.

Les données concernant les densités du rein et du jambon (tabl. 10) informent plus précisément sur les teneurs en différents tissus des morceaux de découpe correspondants : le fait le plus marquant concerne l'augmentation des densités (du rein et du jambon), chez les truies ayant achevé une gestation et ayant reçu, durant cette gestation, le régime NP. Il traduit donc, pour ces animaux, un enrichissement en tissus maigres, ce qui n'est pas observé, par contre, chez les truies non gravides. En lactation, une diminution importante des densités n'est observée que pour les truies du groupe NP. 


\section{Discussion et conclusion}

L’abaissement du taux protéique du régime de gestation (correspondant à la suppression du complément protéique) ne modifie pas le niveau de productivité des truies à la parturition, ce qui confirme le résultat d'un certain nombre d'études résumées précédemment (RERAT \& DuEE, 1975). Toutefois, le maintien, après la mise bas, du régime de gestation le plus riche en protéines $(13,5$ p. 100) crée, au cours de la lactation, un déficit en certains acides aminés indispensables, si l'on se reporte aux données correspondantes obtenues en bilans (Duee, Pastuszewska \& Etienne, 1975). Il s'ensuit une perte de poids prononcée durant cette période, analogue à celle obtenue dans une expérience antérieure (DuEe, 1976). Dans ces conditions, les résultats de l'étude portent essentiellement sur les modifications apportées par la diminution du taux protéique et liées au métabolisme de la mère et à sa composition corporelle au cours d'un cycle de reproduction.

\section{A. Modifications de la composition corporelle après une gestation : effet de la consommation de protéines}

L'ensemble des paramètres étudiés montre une certaine convergence quant à l'effet d'une réduction du taux protéique durant la gestation. La croissance pondérale des mères ainsi que le bilan azoté à 80 jours sont réduits, ce qu'ont déjà montré RIPPEL et al. (1965), Holden et al. (1968), Miller et al. (1969) ou Hawton \& Meade (1971). Parallèlement, les mesures effectuées sur la carcasse des animaux, après parturition, soulignent une diminution des quantités de muscles déposées, confirmant nos précédentes données obtenues 9 jours avant le terme.

Le groupe des truies non gravides est beaucoup moins sensible à cette diminution des apports azotés : ni le bilan azoté, ni la quantité de muscles dans la carcasse n'en sont affectés; seul, le compartiment graisseux diminue, provoquant une légère réduction de la croissance propre des animaux.

Par rapport aux truies vierges, les truies en gestation présentent, au taux protéique le plus bas, un état nutritionnel (croissance nette, bilan azoté, composition corporelle) comparable, ce qui n'est plus vrai au taux protéique le plus élevé. Dans ce cas, la gestation procure un gain de poids supplémentaire ainsi qu'un dépôt musculaire plus important, au détriment des masses graisseuses. En première approximation (d'après les relations établies par Desmoulin, Grandsart \& Tassencourt, 1976), cette augmentation du compartiment musculaire serait d'environ 15 p. 100. Cette modification de la composition corporelle de la truie gravide vers un anabolisme musculaire a déjà été notée par Salmon-Legagneur (1965) et par Elsley et al. (1966). On constate, ici, d'une part qu'elle est fonction du niveau de l'apport protéique durant la gestation et, d'autre part, qu'elle se retrouve aux autres stades d'abattage après parturition (21 jours de lactation, 8 jours après sevrage). Cette propension chez la femelle gravide à déposer préférentiellement des muscles n'a pas toujours été retrouvée chez la truie. Il faut constater toutefois que bien souvent certaines conditions ne sont pas remplies, qui concernent soit l'apport protéique (trop faible chez DE WILDE, 1980 a et b), soit l'état nutritionnel des animaux au moment de la fécondation et leurs potentialités ultérieures de croissance (de Wilde, van Spaendonck \& Vanschoubroek, 1973 ; Lodge, Friend \& Woly- 
NETZ, 1979). On sait, en effet, que la croissance prépubertaire peut affecter les potentialités de dépôt protéique au cours de la gestation (DueE, Treil \& Camous, 1980). Par ailleurs, les observations de Hovell et al. (1977), réalisées 9 jours après le part peuvent masquer l'effet propre de la gestation sur la composition corporelle des animaux. Quoique l'analyse chimique n'ait pas été réalisée, on peut penser que le dépôt accru de muscles implique un dépôt protéique supplémentaire (reflété par le bilan azoté à 80 jours). On admettra, en effet, avec HeAP \& Lodge (1967) que le supplément de protéines déposées dans le compartiment musculaire n'augmente pas leur teneur en eau ; c'est le cas également chez la ratte gestante (LETURQue, communication personnelle).

En définitive, l'état de gestation permet, à la femelle, d'orienter son métabolisme vers un anabolisme protéique (ou une diminution du catabolisme correspondant), à condition que l'apport quantitatif et qualitatif en protéines soit satisfaisant. Ce dernier point ne semble pas être respecté dans le cas du régime à 10 p. 100 de protéines où la teneur en lysine libre du sang s'abaisse nettement, conséquence d'un apport trop modeste en cet acide aminé par rapport aux besoins (DUEE \& RERAT, 1975).

\section{B. Nature de la perte pondérale au cours de la lactation}

$\mathrm{Au}$ cours de la lactation, les animaux doivent faire face à un niveau élevé d'exportation de nutriments dans le lait : pour ce qui concerne les protéines, les quantités évacuées par ce biais s'élèvent à plus de 50 p. 100 des quantités consommées. Cette exigence nutritionnelle explique une mobilisation importante des réserves de l'animal pour couvrir les besoins énergétiques et azotés compatibles avec le niveau de production laitière atteint. La perte de poids au cours de cette période est elle-même fonction de ce niveau de production laitière.

Quand on considère la nature des tissus mobilisés, on met en évidence la prépondérance des masses musculaires. Il existe, en effet, une bonne convergence entre les différents paramètres étudiés. Toutefois, ceci n'exclut pas une mobilisation du tissu adipeux (sous-cutané), au moins dans le cas où les animaux reçoivent en gestation le régime le plus pauvre en protéines. Il est à remarquer que cette mobilisation du tissu adipeux ne s'accompagne pas d'une diminution de la taille des adipocytes, mais l'on peut se demander si le site de prélèvement choisi (cou) est bien représentatif de l'évolution générale : c'est, en effet, à ce niveau que les différences d'épaisseur du lard dorsal sont minimales.

Peu d'études ont porté sur la nature des tissus mobilisés au cours de la lactation. Salmon-Legagneur (1965) a montré le rôle complémentaire des muscles et des masses graisseuses pendant cette période, alors qu'O'GraDY et al. (1975) ne retiennent qu'une diminution des masses adipeuses après trois cycles de reproduction. Dans le cas présent, il faut noter que le catabolisme musculaire caractérisant la perte pondérale de lactation a lieu conjointement avec un bilan azoté faiblement positif et une urémie élevée : il est ainsi vraisemblable que la couverture du besoin énergétique représente, à ce stade, la priorité nutritionnelle.

\section{Adaptation du métabolisme maternel au cours du cycle de reproduction}

Comme nous venons de le souligner, les deux périodes successives (gestation puis lactation) se traduisent par des modifications de la composition corporelle des animaux. 
Ces transformations sont la conséquence des adaptations du métabolisme maternel face aux dépenses correspondant d'une part au développement fœtal, d'autre part à la production laitière. Certains aspects ont été décrits dans cette étude.

Au cours de la gestation, il semble ainsi que le métabolisme du tissu adipeux maternel présente une activité biphasique. A une phase où la lipogénèse est active (jusqu'à 60 jours dans la présente expérience) succède une période caractérisée par une diminution de l'activité de la lipoprotéine lipase du tissu adipeux et par une baisse de la synthèse de novo de lipides. Ces données confirment l'évolution du métabolisme lipidique au cours de la gestation obtenue chez la truie (ETIENNE \& Henry, 1973) ou dans d'autres espèces (Knopp et al., 1973 ; Chilliard et al., 1978 ; Steingrimsdottir, Brasel \& Greenwood, 1980 ; Vernon, ClegG \& Flint, 1981). Confrontés aux résultats de composition corporelle obtenus à la parturition, ces résultats sugggèrent également l'existence d'une lipolyse dans le tissu adipeux en fin de gestation. L'implication de différentes hormones a été soulignée (Kalkhoff, Kissebah \& Kim, 1978). Cette étude montre que l'adaptation du métabolisme maternel est dépendante de l'apport protéique du régime : au plus faible taux de protéines, l'évolution biphasique est moins tranchée et les femelles sont plus grasses à la parturition.

Pour ce qui concerne le métabolisme protéique, cette évolution biphasique a été également avancée, tout au moins chez le rat (NAISMITH, 1980). Nous avons déjà noté que le besoin en certains acides aminés indispensables (lysine, en particulier) est accru en fin de gestation (par rapport aux animaux non gravides). Sur la base des variations de l'aminoacidémie libre, ce besoin semble couvert par l'apport protéique le plus élevé. D'ailleurs, la diminution de l'urémie en fin de gestation, associée à une augmentation de la rétention azotée souligne une meilleure utilisation des protéines alimentaires. Par contre, au taux le plus faible en protéines, la fin de la gestation se caractérise dans ce domaine par l'accroissement de l'urémie correspondant probablement à une protéolyse musculaire qui est corroborée par la diminution de l'azote retenu et des quantités de muscles dans la carcasse.

En lactation, lactivité métabolique du tissu adipeux sous-cutané des mères se maintient à un niveau bas (activité de la lipoprotéine lipase, lipogénèse). On notera cependant que l'activité de la lipoprotéine lipase du tissu adipeux est inversement corrélée avec la production laitière établie à 10 jours de lactation $(\mathrm{r}=-0,57$; $\mathrm{n}=26$ ). Cette relation démontre que l'adaptation du métabolisme maternel au cours de la lactation est dépendante du niveau de production laitière.

En définitive, les résultats de cette expérience ont permis de préciser certaines modifications du métabolisme maternel pouvant induire des transformations dans la composition corporelle des mères. Ces modifications pourraient être liées à des changements de l'état hormonal en gestation comme en lactation mais ce dernier point reste, chez la truie, à préciser. Elles sont, par contre, modulées en fonction de l'apport protéique durant la gestation. 


\section{Summary \\ Effects of a reduced protein supply during gestation on dam metabolism and body composition during the reproductive cycle in the sow}

An experiment was made in 58 Large White gilts distributed at sexual maturity into two groups only differing in the protein supply (10 or 13.5 p. 100 crude protein). Each group included four categories of animals : non pregnant gilts fed in the same conditions as the pregnant gilts and slaughtered 114 days after sexual maturity; gilts mated at sexual maturity and slaughtered within 48 hours after parturition or on day 21 of lactation or 8 days after weaning. After farrowing the animals received the same diet (with 13.5 p. 100 crude protein).

Administration of the diet including 13.5 p. 100 crude protein during gestation increased the gilt weight gain by $16 \mathrm{p} .100$ corresponding to an increase in the net live weight gain (23 p. 100) without any change in litter weight at birth (table 2). In the non-pregnant gilts the weight gain was lower $(11 \mathrm{p}$. 100). Besides, their nitrogen balance, determined after 80 days of experiment, was not changed. Conversely, in pregnant gilts the amount of retained nitrogen increased with the protein supply from 9.3 to $12.0 \mathrm{~g}$ per day (table 4). In late gestation free aminoacidemia in pregnant gilts (table 6) showed an increase in some non essential aminoacids (Asp, Glu, Gly, Ala) associated, at the lowest protein level, with a decrease in the contents of some essential amino acids (Leu, Val, Lys).

The metabolic activity of the subcutaneous fatty tissue (lipoprotein-lipase activity, lipogenesis from glucose) decreased during the second half of gestation (fig. 1 and 2 ). At parturition increase in the protein intake of the pregnant gilts resulted in a higher muscle deposition (Parisian cutting of the carcass, loin and ham density, eye muscle area, backfat thickness). Non-pregnant gilts did not exhibit such a high muscling score (tables 8, 9, 10).

During lactation, the animal performances (milk production, milk composition, litter growth, nitrogen balance) were comparable whatever the protein supply during gestation (10 or 13.5 p. 100) (tables 3 and 5). The metabolic activity of the subcutaneous fatty tissue remained low : an inverse linear relationship was observed between the lipoprotein lipase activity of the subcutaneous fatty tissue and milk production on day 10 of lactation. The weight loss observed during this period mainly resulted in a large mobilization of the muscle masses ( 25 p. 100 reduction of the eye muscle area) (table 9). After weaning, the effect of the protein during gestation on dam body composition persisted, suggesting the existence of a relationship between the level of protein intake during gestation and the nutritional status of the animals after one reproductive cycle.

\section{Références bibliographiques}

Chilliard Y., Durand G., Sauvant D., Morand-Fehr P., 1978. Activité métabolique du tissu adipeux de la chèvre au cours de la gestation et en début de lactation. C.R. Acad. Sc. Paris, 287, 1131-1134.

Desmoulin B., Grandsart P., Tassencourt L., 1976. Les critères d'appréciation de la composition anatomique de la carcasse du porc et des pièces de découpe. Journ. Rech. porcine en France, 8, 89-98.

DueE P.H., 1976. Chronologie de l'apport azoté pendant le cycle de reproduction chez la truie. Ann. Zootech., 25, 199-212.

Duee P.H., Rerat A., 1975. Etude du besoin en lysine de la truie gestante nullipare. $A n n$. Zootech., 24, 447-464. 
Duee P.H., Pastuszewska B., Etienne M., 1975. Utilisation comparée de la féverole et du tourteau de soja par la truie en lactation. II. - Digestibilité et utilisation métabolique des acides aminés. Journ. Rech. porcine en France, 7, 121-128.

DueE P.H., Treil F., Camous S., 1980. Influence de l'apport protéique durant la croissance et la première gestation sur les performances de reproduction et la composition corporelle chez la truie. Ann. Zootech., 29, 121-136.

Elsley F.W.H., Anderson D.M., McDonald I., MacPherson R.M., Smart R., 1966. A comparison of the live-weight changes, nitrogen retention and carcass composition of pregnant and non-pregnant gilts. Anim. Prod., 8, 391-400.

Etienne M., Henry Y., 1973. Influence de l'apport énergétique sur l'utilisation digestive et métabolique des nutriments, et les performances de reproduction, chez la truie gestante nullipare. Ann. Zootech., 22, 311-326.

Hawton J.D., Meade R.J., 1971. Influence of quantity and quality of protein fed the gravid female on reproductive performance and development of offspring in swine. J. Anim. Sci., 32, 88-95.

HeAP F.C., LODGE G.A., 1967. Changes in body composition of the sow during pregnancy. Anim. Prod.., 9, 237-245.

Holden P.J., Lucas E.W., Speer V.G., Hays V.W., 1968. Effect of protein level during pregnancy and lactation on reproductive performance of sows. J. Anim. Sci., 27, $1587-1590$.

Hovell F.D.B., MacPherson R.M., Crofts R.M.J., Smart R.I., 1977. The effect of pregnancy, energy intake and mating weight on protein deposition and energy retention of female pigs. Anim. Prod., 25, 281-290.

Kalkhoff R.K., Kissebah A.H., Kim H.J., 1978. Carbohydrate and lipid metabolism during normal pregnancy : relationship to gestational hormone action. Semin. Perinatal., 2, 291-307.

Knopp R.H., Saudek C.D., ArKy R.A., O'Sullivan J.B., 1973. Two phases of adipose tissue metabolism in pregnancy : maternal adaptations for fetal growth. Endocrinology, 92, 984-988.

Lodge G.A., Friend D.W., Wolynetz M.S., 1979. Effect of pregnancy on body composition and energy balance of the gilt. Can. J. Anim. Sci., 59, 51-61.

Miller G.M., Becker D.E., Jensen A.H., Harmon B.G., Norton H.W., 1969. Effect of protein intake on nitrogen retention by swine during late pregnancy. J. Anim. Sci., 28, 204-207.

Naismith D.J., 1980. Maternal nutrition and the outcome of pregnancy - a critical appraisal. Proc. Nutr. Soc., 39, 1-11.

O'Grady J.F., Elsley F.W.H., MacPherson R.M., McDonald I., 1975. The response of lactating sows and their litters to different dietary energy allowances. Anim. Prod., 20, 257-265.

O'Hea E.K., LeVeille G.A., 1968. Lipogenesis in isolated adipose tissue of the domestic chick (Gallus domesticus). Comp. Biochem. Physiol., 26, 1081-1089.

Pascal. G., Durand G., Macaire J.P., Penot E., 1977. Evolution de l'activité de l'acétylCoA-carboxylase et de la lipoprotéine lipase dans le foie et les tissus adipeux du rat mâle au cours du développement après sevrage. Influence du taux de lipides dans la ration. Ann. Biol. anim. Biochim. Biophys., 17, 827-849.

Rerat A., DueE P.H., 1975. Ernährung und Reproduktion der Sau. Ubers. Tierernährg., 3, 101-141, 249-276.

Rippel R.H., Rasmussen O.G., Jensen A.H., Norton H.W., Becker D.E., 1965. Effect of level and source of protein on reproductive performance of swine. J. Anim. Sci., 24, 203-208. 
Rodbell M., 1964. Metabolism of isolated fat cells. Effects of hormones on glucose metabolism and lipolysis. J. Biol. Chem., 239, 375-380.

Salmon-Legagneur E., 1956. La mesure de la production laitière chez la truie. Ann. Zootech., 5, 95-110.

Salmon-Legagneur E., 1965. Quelques aspects des relations nutritionnelles entre la gestation et la lactation chez la truie. Ann. Zootech., 14, $n^{\circ}$ H.S. 1, 1-137.

Steingrimsdottir L., Brasel J.A., Greenwood M.R.C., 1980. Diet, pregnancy and lactation : effects on adipose tissue, lipoprotein lipase, and fat cell size. Metabolism, 29, 837-841.

Vernon R.G., Clegg R.A., Flint D.J., 1981. Metabolism of sheep adipose tissue during pregnancy and lactation. Biochem. J., 200, 307-314.

De Wilde R., Van Spaendonck R., Vanschoubroek F., 1973. Energy retention of pregnant and non pregnant gilts. Proc. 6th Symp. on energy metabolism of farm animals. E.A.A.P., Stuttgart.

DE WILDE R.O., 1980 a. Protein and energy retentions in pregnant and non pregnant gilts. 1. - Protein retention. Livest. Prod. Sci., 7, 497-504.

DE WILdE R.O., 1980 b. Protein and energy retentions in pregnant and non-pregnant gilts. 2. - Energy retention. Livest. Prod. Sci., 7, 505-510. 